Apidologie, 1972, 3 (4) 341-367.

\title{
ÉCOLOGIE DE QUELQUES TRIGONES (TRIGONA SP.) DE LA SAVANE DE LAMTO (COTE D'IVOIRE)*
}

\author{
Ökologie einiger Trionen (Trigona Sp.) \\ in der Savanne von Lamto (Elfenbeinküste)
}

\author{
Roger DARCHEN \\ Faculté des Sciences de Paris, Station Biologique des Eyzies \\ 24 - Les Eyzies \\ et Station d'Écologie Tropicale de Lamto, B.P. 28 à N'Douci \\ Côte d'Ivoire
}

\begin{abstract}
SUMMARY
THE ECOLOGICAL ROLE OF THE TRIgONES (Trigonini)

IN THE SAVANNAH AROUND LAMTO, IVORY COAST
\end{abstract}

This study attemps to clarify the ecological role of social bees, in particular the Trigones (Trigonini) in the savannah around Lamto, Ivory Coast.

1) Over an area of 30 hectares $\left(300000 \mathrm{~m}^{2}\right)$ made up of quadrats of various sizes an average of 2.5 nests per hectare were found. This contrasts with LEVIEUX's estimate of 7,000 ant's nests per hectare, and Josens'density estimate of 17 million termites per hectare.

2) The small bee species (Hypotrigona and Liotrigona) appear to show no biotopic preference for any particular feature of savannah.

3) The development of the Hypotrigona workers lasts from 39 to 40 days.

4) The number of eggs produced daily by a queen varies somewhat from one colony to another (from 22 to 82 eggs per day).

5) The rate of egg production is independent of the number of individuals in the colony.

6) At Lamto, the monthly average weight of the brood stays almost the same during the wet and the dry seasons.

7) The estimated weight of nymphs, larvae and food in an average colony of 1,100 individuals weighing $3.3 \mathrm{~g}$ is $5 \mathrm{~g}$. The weight of the colony is thus $8.3 \mathrm{~g}$. Thus, with 2.5 nests, the average weight per hectare is $21 \mathrm{~g}$.

8) There is a notable reduction in food reserves from the wet season to the first half

* Ce travail a été effectué au laboratoire d'Écologie tropicale de Lamto dans le cadre de la R.C.P. 60 du C.N.R.S. et du P.B.I. 
of the dry season : the average food reserve of the Hypotrigones per hectare is around $820 \mathrm{~g}$. in October and $50 \mathrm{~g}$ in May.

How relatively unimportant seem the 2,750 Hypotrigones found per hectare, when compared to the biomass of Cerambycids or worms, or even other social insects. Yet the bees are of capital importance for polliniation given that there may be 800 flights daily per colony of Hypotrigones. That is to say 2,000 daily foraging flights over one hectare of savannah.

\section{RÉSUMÉ}

Ce travail tente d'éclaircir le rôle écologique des abeilles sociales, en particulier des Trigones, dans la savane de Lamto, Côte d'Ivoire.

1) Sur les 30 hectares, divisés en quadrats de surfaces diverses, nous avons trouvé une moyenne de 2,5 nids à l'hectare. (Il y a 7000 nids de Fourmis d'après LeviEux (1969) et pour les Termites 17 millions d'individus d'après Josens (1969) sur un hectare). Certaines zones en sont complètement dépourvues alors que d'autres en contiennent plusieurs.

2) Aucun faciès de savane ne semble être le biotope préférentiel pour l'établissement des petites espèces d'abeilles (Hypotrigones et Liotrigones).

3) Le développement de l'ouvrière des Hypotrigones dure de 39 à 40 jours.

4) La ponte journalière d'une reine est assez variable d'une colonie à une autre : elle va de 22 cufs à 82 .

5) Le taux de ponte est indépendant du nombre d'individus présents dans la colonie.

6) A Lamto, la moyenne mensuelle des poids de couvain, estimée en grammes, reste à peu près la même durant la saison des pluies et la saison sèche.

7) On peut estimer à environ $5 \mathrm{~g}$ le poids des nymphes, des larves et de leur nourriture dans une colonie moyenne de 1100 individus pesant $3,3 \mathrm{~g}$. Le poids moyen d'une colonie est donc environ $8,3 \mathrm{~g}$, celui des 2,5 colonies à l'hectare de $21 \mathrm{~g}$.

8) Il y a une diminution notable des réserves de la saison des pluies à la saison sèche bien entamée. La moyenne des réserves des Hypotrigones sur un hectare est d'environ $820 \mathrm{~g}$ au mois d'octobre et $50 \mathrm{~g}$ en mai.

Que sont 2750 Hypotrigones à l'hectare de savane en face de la biomasse des Cérambycides, des Vers ou même d'autres insectes sociaux? Presque rien. Pourtant l'importance des abeilles est capitale pour la pollinisation puisqu'il peut y avoir environ 800 vols journaliers d'Hypotrigones par colonie, c'est-à-dire 2000 vols de butinage au-dessus d'un hectare de savane.

\section{I. - INTRODUCTION}

Notre travail entre dans le cadre du Programme de Biologie International. Il a pour but d'éclaircir le rôle écologique des abeilles sociales, en particulier des abeilles sans dard ou Trigones, dans la savanne de Lamto, Côte d'Ivoire.

La station de recherches d'écologie tropicale de Lamto se trouve à environ $200 \mathrm{~km}$ au nord-est de la ville d'Abidjan dans une savane de type guinéen à palmiers rôniers (Borassus aethiopum). Cette savane s'enfonce comme un coin à l'intérieur de zones de forêt dense tropicale longeant l'océan Atlantique, aussi est-il classique d'appeler cette avancée le $\mathrm{V}$ baoulé. Elle a les coordonnées suivantes : $5^{\circ} 02^{\prime}$ de longitude ouest, $6^{\circ} 13^{\prime}$ de latitude nord (fig. 1 ).

La savane est essentiellement composée d'un tapis graminéen d'où 
émergent des arbres et parfois des buissons. L'architecture de ces végétaux ligneux conditionne les possibilités du peuplement par les abeilles. Le nombre des espèces ligneuses est limité et nous pouvons ici les classer en deux catégories :

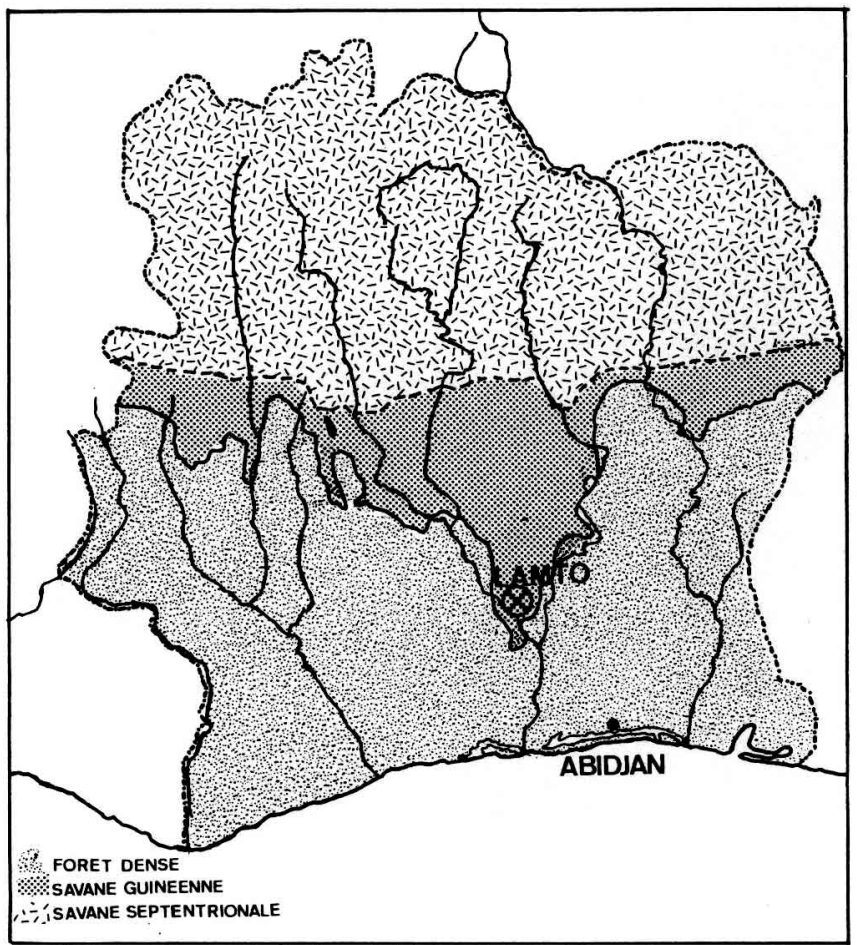

Fig. 1. - Carte Phytogéographique de la Côte d'Ivoire. Авв. 1. - Phytogeographische Karte der Elfenbeinkiiste.

\section{1. - Les arbres non utilisés pour la nidification des abeilles}

Il s'agit essentiellement du Palmier rônier et de Cussonia barteri. Nous avons rencontré une fois, dans un rônier mort, une colonie d'Apis mellifica et une autre fois un nid de Dactylurina accolé au haut d'un tronc de rônier vivant (fig. 2) mais ces deux trouvailles sont restées exceptionnelles et nous devons considérer le rônier comme non favorable à la nidification des abeilles sociales. L'araliacée, Cussonia barteri ne recèle jamais d'abeilles. Cela tient à la structure de la plante. Une coupe transversale dans une branche montre que cet arbre très particulier est revêtu d'une écorce subéreuse très épaisse, crevassée longitudinalement, et entourant un cœur très tendre, gorgé d'une 
abondante sève. Il est impossible aux abeilles de coloniser les parties vivantes de ce végétal. Quant aux parties mortes, elles ne peuvent être utilisées non

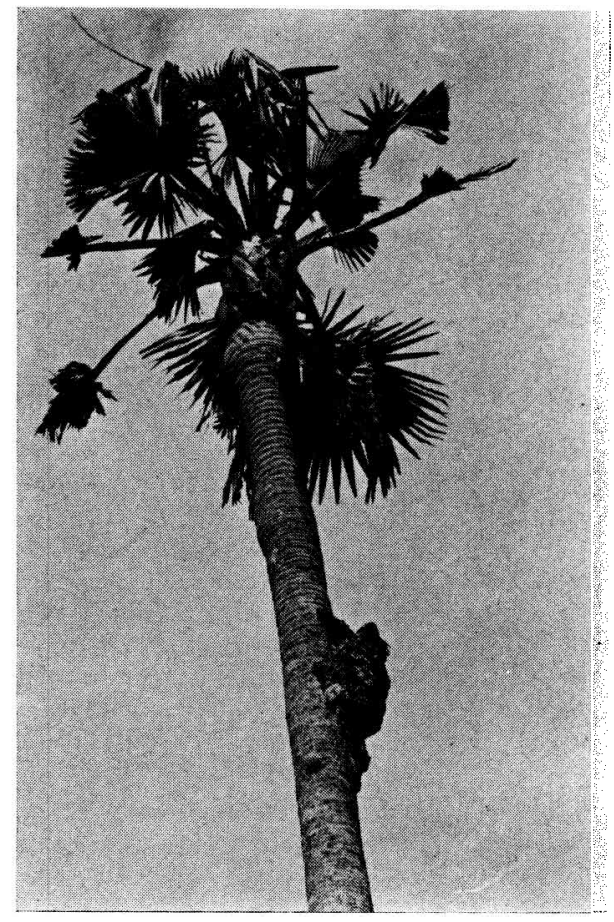
plus, car dans ce cas le bois peu lignifié est vite réduit en poussière; seule persiste un certain temps l'écorce, mais dans l'ensemble elle demeure fragile et une occupation de longue durée n'est guère possible.

FIG. 2. - Nid de Dactylurina accrochê à un palmier. Ces abeilles construisent leur nid de toutes pièces en utilisant de la cire, de la résine, des dêchets végétaux.

AвB. 2. - Nest von Dactylurina, an einer Palme befestigt. Diese Bienen errichten vollkommen freigebaute Nester, wobei sie Harz, Wachs und pflanzlichen Abfall verwenden.

2. — Les arbres utilisés par les abeilles pour leur nidification

Ce sont essentiellement tous les arbres présentant des cavités internes. On peut citer parmi les essences les plus répandues : Crossopteryx febrifuga, Bridelia ferruginea, Piliostigma thonningii, Terminalia glaucescens, Vitex doniana (fig. 3 et 4). Les causes de l'évidement interne de ces arbres sont assez obscures et vraisemblablement complexes. Les champignons, les Insectes (Coléoptères, Termites, Fourmis peut-être) et même le feu doivent concourir à cette dégradation. De toute façon, ces bois sont dans l'ensemble très durs et il est exclu que les abeilles puissent les entamer pour aménager leur habitation. Dans tous les cas, elles profitent de cavités préexistantes. Dès lors qu'un arbre a le tronc ou les branches crevassés il est susceptible d'être habité par des abeilles sociales, qu'il soit mort ou vivant (fig. 5). Les abeilles utilisent la cavité sans tenir compte des avantages thermorégulateurs ou hygrorégulateurs que pourraient leur apporter le bois vivant. Elles sont adaptées à la savane et capables de réguler leur propre micro-climat. C'est là une différence 
essentielle avec les Fourmis (Crematogaster) qui logent toujours dans la partie vivante des arbres (B. Delage-Darchen, 1971).

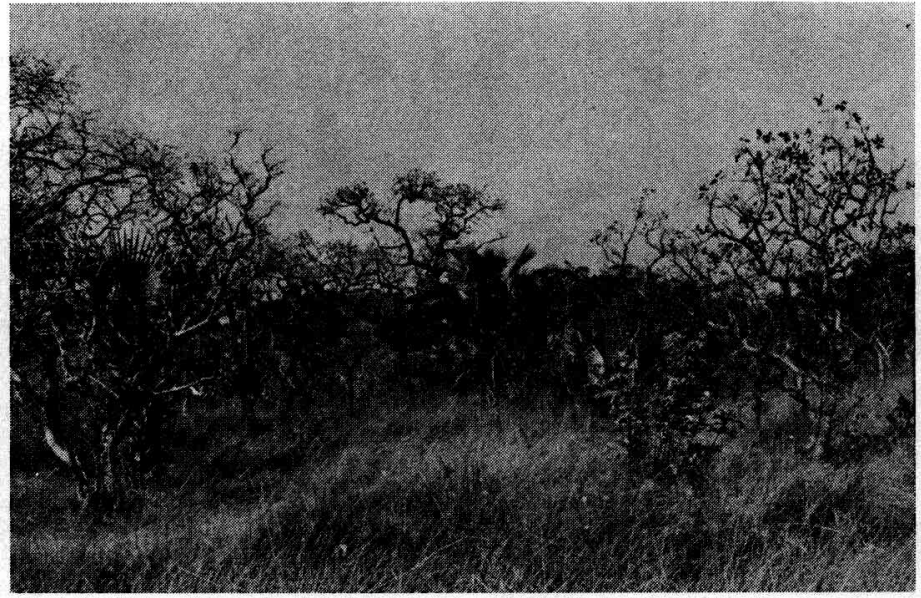

FiG. 3. - Faciès de savane arbustive dense à Andropogonées.

Авв. 3. - Dicht mit Andropogonen bewachsene und mit Strauchwerk bestandene Savanne.

Fig. 4. - Un des nombreux Crossopteryx creux contenant un nid d'Abeilles sociales (Hypotrigones ou Liotrigones).

Les troncs d'arbres ont en général au minimum dix centimètres de diamètre.

Авв. 4. - Einer der zahlreichen hohlen Crossopteryx mit einem Nest sozialer Insekten (Hypotrigona oder Liotrigona).

Die Baumstämme haben im allgemeinen einen Durchmesser von mindestens $10 \mathrm{~cm}$.

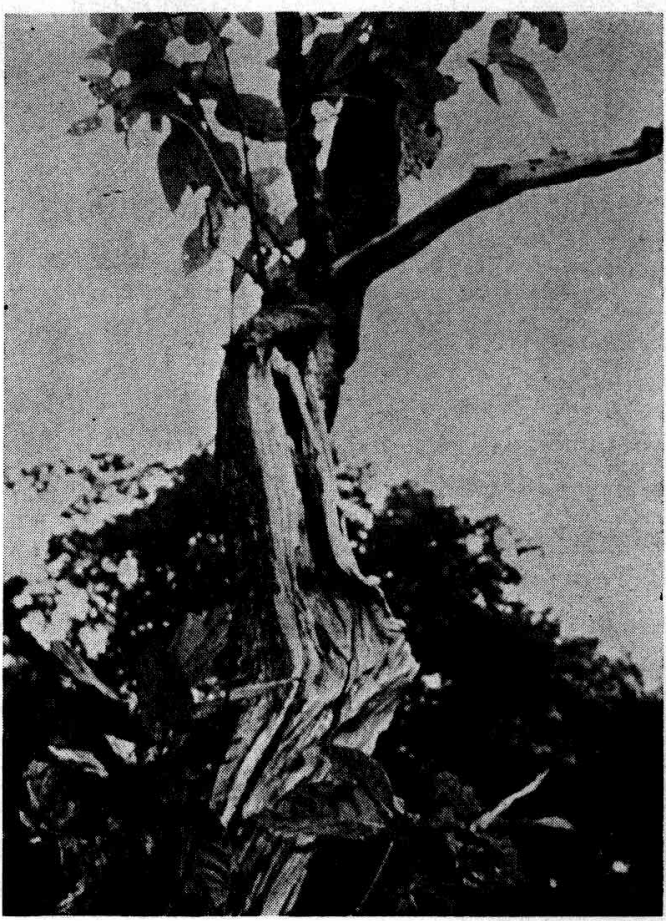


Mais il existe aussi des abeilles de savane qui ne logent pas dans les cavités des arbres. On peut les classer en deux catégories :

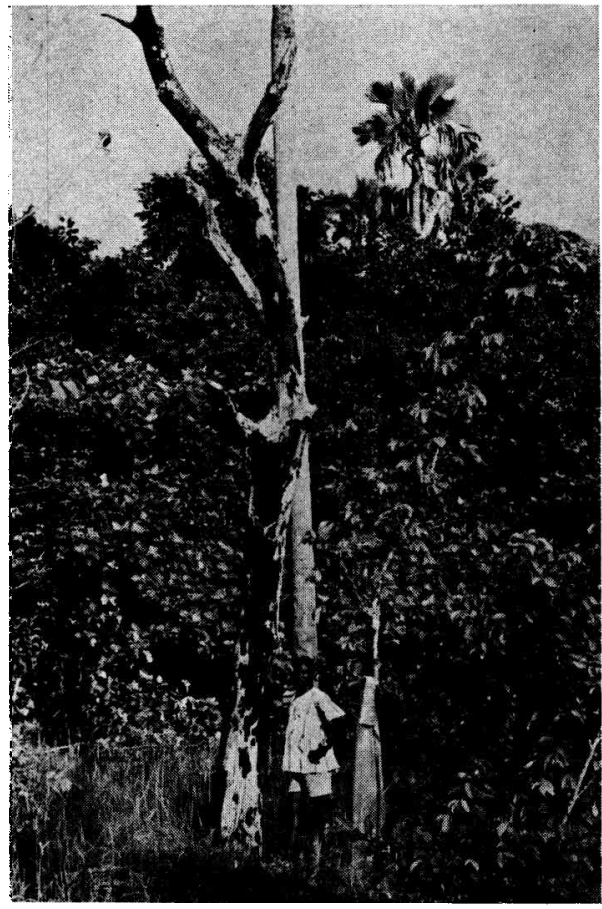

FIG. 5. - Arbre mort et creux, reste d'une forêt galerie dégradée, contenant plusieurs nids d'Abeilles (Axestotrigones et Hypotrigones).

Aвв. 5. - Abgestorbener, hohler Baum, Überrest eines zugrunde gegangenen Galeriewaldes. Er beherbergt mehrere Bienennester. (Axestotrigona und Hypotrigona).

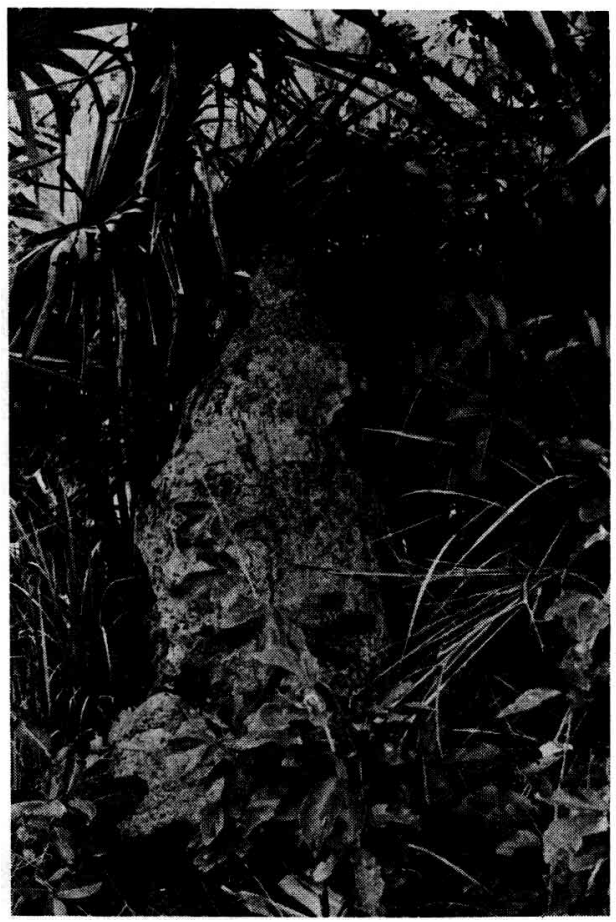

FIG. 6. - Ancienne termitière de Bellicositermes abandonnée et réoccupée par plusieurs espèces de Termites, de Fourmis et d'Abeilles.

Авв. 6. - Alter, verlassener Termitenhügel von Bellicositermes, der von mehreren Termitenarten, Ameisen und Bienen neu besetzt wurde.

1) Celles qui construisent de toutes pièces des nids en forme de boules suspendues aux branches : elles appartiennent au genre Dactylurina et 2) celles qui utilisent des cavités ménagées dans de grosses termitières (fig. 6). (Darchen, 1969 et 1970). Le problème de l'installation des abeilles dans les Termitières est assez complexe. Signalons tout $d$ 'abord que les Termitières vivantes, c'est-à-dire les Termitières habitées par les véritables constructeurs, ne sont jamais colonisées par les abeilles. Cela peut se comprendre car, dans ce cas, la Termitière est un bloc compact, toutes les effractions éventuelles sont vite colmatées par les ouvriers et les ouvertures sont gardées par des soldats agressifs. L'intrusion des abeilles est donc impossible. Par contre, lorsque la grosse Termitière est morte, elle peut être réoccupée par d'autres espèces de Termites plus petites qui cohahitent dans ce bloc cloisonné en 
s'ignorant. Il arrive alors que des brèches soient ouvertes dans ces Termitières réoccupées. Les agents destructeurs ne sont pas toujours faciles à identifier. Il est vraisemblable que Rongeurs, Pangolins ou même Insectes y contribuent (Darchen, 1970). Ce sont ces cavités qui seront par la suite réutilisées par les abeilles. Notons enfin que nous n'avons jamais trouvé de nids d'abeilles dans des Termitières dont la taille soit inférieure à $95 \mathrm{~cm}$ de haut et $100 \mathrm{~cm}$ de diamètre (à la surface du sol). Il faut donc que la Termitière soit assez grosse. Ces constructions sont assez rares dans la réserve de Lamto et la majorité ne contient pas d'abeilles, c'est-à-dire que les espèces vivant dans les Termitières ont été trouvées très peu souvent. Sur plusieurs centaines d'hectares prospectés, Josens (Termitologue) (1969) et moi-même n'en avons rencontré qu'une demi-douzaine de fois.

A notre grand étonnement, nous n'avons jamais découvert de nids d'abeilles creusés profondément dans la terre comme l'on en peut voir au Gabon. Peut-être y en a-t-il dans les forêts galeries ? Jusqu'ici nos recherches ont été vaines, mais il est vrai que ces nids sont très difficiles à repérer.

La savane offre donc aux abeilles différents types de refuges, mais elle les attire encore par de nombreuses plantes et surtout les graminées très recherchées pour le pollen. L'action pollinisatrice des abeilles de savane est certainement très importante et toute étude de ces Insectes doit nécessairement envisager cette question. Nous la réservons pour un autre mémoire et nous n'étudierons ici que les faits intéressants les problèmes de densité et de renouvellement des populations. Lorsque cela nous sera possible, nous tenterons quelques comparaisons entre les faits de biologie que nous connaissons chez les abeilles de savane et chez les abeilles de forêt que nous étudions parallèlement au Gabon.

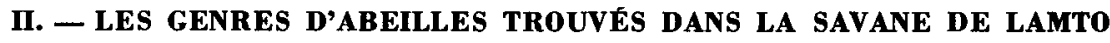

Tous les genres de Trigones découverts en Afrique n'ont pas été retrouvés dans la savane de Lamto. Sur les 10 genres actuellement reconnus en Afrique nous n'avons récolté des représentants que de cinq d'entre eux seulement, soit une quinzaine d'espèces au maximum. L'analyse des échantillons nous permet de penser que 3 ou 4 espèces, au moins, sont nouvelles (un travail plus précis de taxinomie devra être envisagé à la suite de cette étude pour identifier avec rigueur ce matériel difficile où beaucoup d'espèces sont morphologiquement très voisines). La plupart des nids des espèces récoltées ont pu être trouvés et analysés. Ils ont été retirés des arbres creux ou des termitières.

Cependant, il est des espèces que nous avons capturées en savane où elles butinaient, mais qui ne nidifient pas dans cette formation. Elles provenaient 
des forêts galeries toujours proches. C'est le cas par exemple d'Apis mellifica la plus grosse espèce d'abeille qu'on rencontre fréquemment sur les plantes de la savane mais dont le nid est toujours en forêt. Une fois seulement nous avons trouvé une colonie de cette espèce dans la cavité d'un rônier mort en savane, mais le cas est très exceptionnel.

Par contre, toutes les Hypotrigones, toutes les Liotrigones toutes les Dactylurines que nous avons récoltées en savane y établissent leur nid. Elles sont capables de supporter de très fortes insolations et le passage annuel des feux. Quant aux Axestotrigones butinant en savane, certaines sont des formes forestières mais d'autres sont aussi de véritables savanicoles.

Nous avons cherché à savoir comment ces différentes abeilles procèdent pour assurer la thermorégulation de leur nid.

Les Hypotrigones semblent n'utiliser qu'une méthode assez simple; elles s'installent à la queue-leu-leu dans le tube de cire servant de canal d'entrée au nid et elles battent des ailes la tête tournée vers l'extérieur pour assurer un courant d'air rafraîchissant. On peut les observer grâce à la transparence du tube de cire. Les vibrations simultanées produites ainsi par un certain nombre d'insectes engendrent un bruit nettement audible à l'extérieur de la ruche.

Les Dactylurines, elles, disposent de plusieurs moyens pour lutter contre les excès de température. Rappelons que leur nid se présente sous la forme d'une boule accrochée aux branches. Il s'agit donc d'un nid construit de toutes pièces par les Insectes. Au Gabon, ces nids sont très souvent bâtis dans la fourche des branches de cacaoyers ou de caféiers. Ils sont ainsi à l'abri du soleil sous les frondaisons. En Côte d'Ivoire ces abeilles suspendent leur habitation assez haut dans les arbres, en situation peu abritée, contrairement aux espèces gabonaises. On peut les trouver en savane sur les arbres généralement les plus hauts (nous avons même trouvé un nid collé au tronc lisse d'un palmier rônier, donc exposé à toutes les intempéries), mais on les rencontre aussi en forêt galerie; dans ces cas ils sont suspendus aux branches des très grands arbres (Kapokiers par exemple) à plusieurs dizaines de mètre de hauteur : ils sont donc là aussi en position dégagée. Ces abeilles peuvent supporter les excès de température auxquels elles sont soumises grâce à plusieurs artifices :

1) D'abord la structure même du nid entre en ligne de compte. En Côte d'Ivoire les nids sont très volumineux (70 à $80 \mathrm{~cm}$ de diamètre) comparés à ceux du Gabon $(40$ à $50 \mathrm{~cm}$ ). Cela tient surtout à ce que la coque protectrice du nid est très épaisse. Cette coque est constituée d'un mélange de cire, de résine prélevée sur les plantes, et aussi de débris de végétaux. La paroi extérieure est relativement unie mais l'intérieur de la coque est spongieux. De 
nombreuses lames de cette composition cire-résine, certainement mauvaise conductrice de la chaleur, emprisonnent de l'air dans les cavités qu'elles circonscrivent. Ainsi, une première isolation thermique est obtenue par ce moyen.

2) A part ce moyen passif de thermorégulation inhérent à la structure du nid, les Dactylurines utilisent aussi des méthodes actives :

a) elles ventilent,

b) elles apportent des modifications temporaires à l'enveloppe même du nid. Pour cela, elles s'y prennent de deux manières :

- d'une part, elles ouvrent un gros orifice identique à l'orifice d'entrée du nid et situé lui aussi sur le côté et vers le bas mais à l'opposé du trou d'entrée. Une circulation d'air peut ainsi être établie.

- d'autre part, ux heures chaudes elles ouvrent sur toute la surface du nid, dans l'enveloppe extérieure, de nombreux petits trous facilitant encore ainsi la ventilation. Ces ouvertures sont refermées lorsque la température s'abaisse.

Nous ignorons les moyens employés par les Axestotrigones pour maintenir l'homéothermie de leurs ruches. (Rappelons qu'en savane ces abeilles nichent dans les termitières, elles sont donc protégées du rayonnement direct par une épaisseur de terre alvéolée). Il est cependant vraisemblable de penser qu'elles utilisent aussi la ventilation.

\section{III. - ACTION DES FEUX DE BROUSSE SUR LES ABEILLES}

Les abeilles qui sont manifestement le plus exposées aux feux de brousse sont les petites espèces logeant dans les branches des arbres de la savane. Nous avons cherché à évaluer l'action nocive de la chaleur en ouvrant des colonies juste après le passage des feux. Il s'est avéré que bien des nids restent indemnes malgré l'incendie. Mais, lorsque la force du feu est entretenue par des broussailles épaisses au pied d'un arbre où logent des abeilles, on peut constater des dégâts dans les nids. Lorsque le point de fusion de la cire est atteint ou dépassé les cellules fondent et le jeune couvain enfermé dans des coques individuelles de cire est voué à mourir emprisonné alors dans un magma désorganisé (fig. 7). Il y a ainsi au moment des feux des trous dans le renouvellement de la population de certaines colonies, mais cela n'entraîne pas pour autant leur disparition car la reine et bon nombre d'ouvrières restent vivantes.

Nous avons cherché à connaître l'importance des dégâts causés chez les abeilles par les feux de brousse en 1970. Cette année là, les feux ont été malheureusement assez peu fournis. Sur une surface d'un hectare, particulièrement 


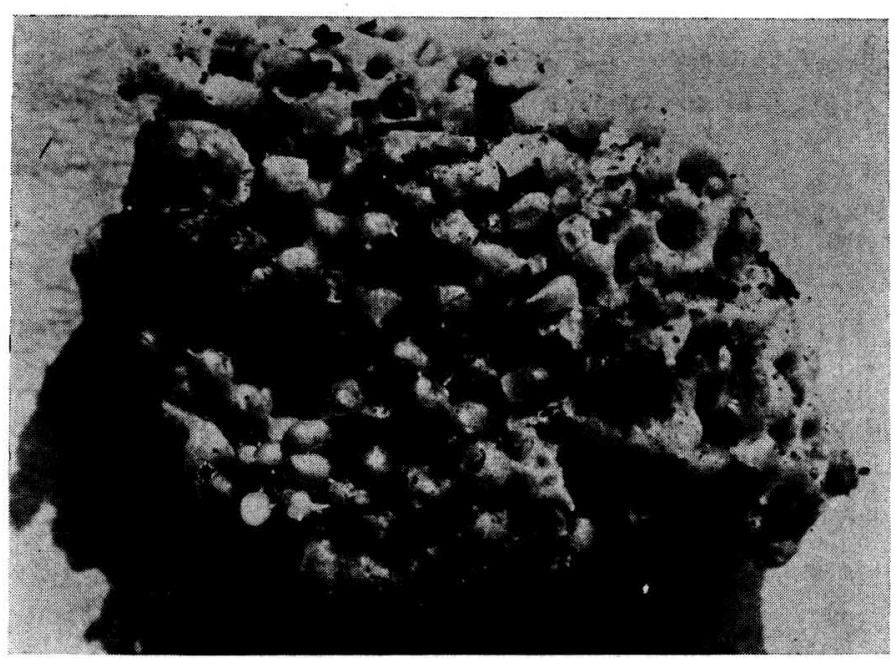

Fig. 7. - Cellules de réserves de pollen ou de miel (à gauche) et cellules de couvain (à droite) fondues après le passage du feu de savane.

Авв. 7. - Vorratszellen mit Pollen und Honig (links) und Brutzellen (rechts), die beim Durchgang des Savannenbrandes geschmolzen sind.

riche en petites espèces (Hypotrigones, Liotrigones) nous avons compté 15 nids. Sur ces 15 nids, deux seulement avaient le couvain endommagé et la cire fondue, mais la population adulte était intacte. Il est probable que la fumée protège les Insectes en les immobilisant à l'intérieur du nid.

Est-ce à dire que le feu n'a aucune action indirecte sur les abeilles ? Certainement non, car la destruction de l'herbe entraîne la disparition d'une source de nourriture. Les animaux doivent alors parcourir de beaucoup plus grandes distances pour aller s'approvisionner. Toutefois ce handicap n'est pas insurmontable pour les petites espèces puisque, à certaines époques, on peut trouver des Hypotrigones sur les inflorescences mâles des rôniers à 15 mètres de haut. Lorsque les conditions l'exigent ces abeilles sont donc capables d'accroître considérablement l'importance de leurs déplacements. Nous avons remarqué en ouvrant des nids d'Hypotrigones en saison sèche une diminution notable des réserves. Il est probable que cette période de sécheresse dont la pauvreté est accentuée encore par le balayage des feux, entraîne périodiquement la diminution et des réserves et du couvain, mais seuls des élevages expérimentaux d'assez longue durée pourront donner une idée précise du phénomène.

\section{IV. - ETUde DE LA DENSITE des POPULATIONS D'ABEILLES}

Pour évaluer la densité des abeilles de savane par unité de surface on a utilisé une technique très classique de dénombrement des nids dans des carrés 
de surface connue, choisis au hasard. Nous avons commencé par délimiter des carrés de 10 mètres, puis 25 et 30 mètres de côté, mais très rapidement nous nous sommes aperçu que les surfaces ainsi délimitées étaient nettement trop petites, la densité des nids étant trop faible pour qu'on ait la chance d'en rencontrer sur de tels échantillons. Aussi avons nous préféré rechercher les abeilles sur des surfaces de $1 / 8$ d'hectare $(50 \mathrm{~m} \times 25 \mathrm{~m})$, d'1/4 d'hectare $(50 \mathrm{~m} \times 50 \mathrm{~m})$ ou même d'l hectare $(100 \mathrm{~m} \times 100 \mathrm{~m})$.

Bien que les emplacements des quadrats aient été déterminés au hasard, nous avons cherché à faire des relevés en nombre comparables dans les différents faciès de la savane, faciès héliophile, arbustifs clairs, arbustifs denses et enfin très boisés.

Dans les zones ainsi étudiées nous avons dénombré les arbres et arbustes de manière à établir les relations possibles entre le nombre d'arbres par unité de surface et le nombre de nid d'abeilles au même endroit. Une première observation se dégage de ce travail : seuls les arbres dont le diamètre du tronc dépasse une dizaine de centimètres ont des chances de posséder des abeilles. En dessous de cette taille ils sont trop petits pour posséder dans le tronc ou les branches maîtresses des cavités internes susceptibles d'héberger les abeilles.

\section{V. - ANALYSE DES RÉSULTATS CONCERNANT LA DENSITÉ DES NIDS D'ABEILLES DANS LA SAVANE DE LAMTO ET DE SES ENVIRONS}

$287500 \mathrm{~m}^{2}$, soit environ 30 hectares, ont été parcourus de long en large en 5 mois de séjour sur la réserve ou dans ses environs par deux ou trois personnes à la recherche des nids d'abeilles. On a ainsi délimité 1 carré de $100 \mathrm{~m}^{2}$, 3 de $500 \mathrm{~m}^{2}, 4$ de $625 \mathrm{~m}^{2}, 1$ de $3000 \mathrm{~m}^{2}, 1$ de $3600 \mathrm{~m}^{2}, 5$ de $10000 \mathrm{~m}^{2}, 2$ de $20000 \mathrm{~m}^{2}$, et un de $30000 \mathrm{~m}^{2}$. Nos résultats portent donc sur 94 quadrats.

Le nombre des nids trouvés sur l'ensemble de ces surfaces s'élève à 72 . Ce qui donne environ une moyenne de 2,5 nids à l'hectare. Ce chiffre nous indique la pauvreté de la faune en abeilles sociales de la savane de Lamto comparée à celle des Fourmis terricoles (7000 nids à l'hectare d'après Levieux, 1969) ou des Termites (17 millions d'individus à l'hectare d'après Josens, 1969). Remarquons immédiatement que ces chiffres ne donnent aucune idée sur la répartition réelle des colonies d'abeilles sur le territoire, certaines zones en sont presque complètement dépourvues alors que d'autres contiennent plusieurs nids.

Le tableau 1 peut seul nous donner un aperçu exact de la distribution des nids dans la réserve.

A la lecture de ce tableau, $1^{\circ}$ ) on voit qu'il n'y a apparemment aucun faciès de savane qui semble être le biotope préférentiel pour l'établissement 
TABLEaU 1

Distribution des nids dans divers faciès de la savane de Lamto

TABeLLE 1

Verteilung der Nester in verschiedenen Savanneabschnitten von Lamto

\begin{tabular}{|c|c|c|c|c|c|}
\hline 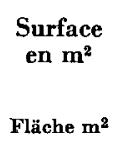 & $\begin{array}{c}\text { Nombre } \\
\text { d'arbres à } \\
\text { l'hectare } \\
\text { Zahl der } \\
\text { Bäume je Ha }\end{array}$ & $\begin{array}{c}\text { Nombre } \\
\text { de nids } \\
\text { Zahl der } \\
\text { Nester }\end{array}$ & $\begin{array}{c}\begin{array}{c}\text { Surface } \\
\text { en } \mathrm{m}^{2}\end{array} \\
\text { Fläche } \mathrm{m}^{2}\end{array}$ & $\begin{array}{c}\text { Nombre } \\
\text { d'arbres à } \\
\text { l'hectare } \\
\text { Zahl der } \\
\text { Bäume je Ha }\end{array}$ & $\begin{array}{c}\text { Nombre } \\
\text { de nids } \\
\text { Zahl der } \\
\text { Nester }\end{array}$ \\
\hline $\begin{array}{rr}10 & 000 \\
30000 \\
20000 \\
107500 \\
18000 \\
10000 \\
10000 \\
10000 \\
2500 \\
2500 \\
3000 \\
2500 \\
625 \\
625\end{array}$ & $\begin{array}{r}340 \\
900 \\
\\
222 \\
222 \\
82 \\
95 \\
55 \\
160 \\
148 \\
\\
350 \\
500 \\
500\end{array}$ & $\begin{array}{r}2 \\
8 \\
3 \\
25 \\
6 \\
0 \\
2 \\
0 \\
0 \\
1 \\
0 \\
3 \\
0 \\
2\end{array}$ & $\begin{array}{rr} & 625 \\
& 625 \\
3 & 600 \\
1 & 250 \\
2 & 500 \\
2 & 500 \\
2 & 500 \\
2 & 500 \\
2 & 500 \\
2 & 500 \\
& 500 \\
& 500 \\
& 500\end{array}$ & $\begin{array}{r}560 \\
962 \\
33 \\
96 \\
192 \\
272 \\
205 \\
160 \\
340 \\
212 \\
860 \\
340 \\
800\end{array}$ & $\begin{array}{c}0 \\
25 \text { dont } 22 \\
\text { dans le } \\
\text { même arbre } \\
0 \\
1 \\
0 \\
0 \\
0 \\
0 \\
2 \\
7 \\
3 \\
3 \\
1 \\
2 \\
0\end{array}$ \\
\hline
\end{tabular}

des petites espèces d'abeilles (Hypotrigones et Liotrigones) ou des espèces moyennes comme Trigona sawadogoi ou Trigona eburnensis : tous les faciès cités plus haut, à condition qu'ils possèdent des arbres creux, c'est-à-dire des arbres ayant en général plus de $10 \mathrm{~cm}$ de diamètre, ou des Termitières abandonnées, sont susceptibles d'abriter des abeilles. On fera une exception pour Dactylurina qui n'a été trouvée que dans des savanes fermées à proximité de forêts galeries. $2^{\circ}$ ) Ce tableau nous montre aussi l'irrégularité de la dispersion des nids sur toute l'étendue de la réserve : des hectares entiers peuvent être dénués de colonie d'abeilles tandis que d'autres en contiennent plusieurs. Ce phénomène n'est pas étonnant quand on connaît la biologie des petites espèces d'abeilles et leur essaimage en particulier. En effet, les exploratrices d'une colonie prête à essaimer recherchent toujours une habitation à proximité de la souche mère : elles la trouvent quelquefois à quelques centimètres, à quelques mètres, rarement beaucoup plus loin. Les conditions d'essaimage exigent presque cette proximité car les relations entre la colonie ancienne et la nouvelle peuvent durer quelquefois plusieurs semaines : la colonisation du nouvel habitat se fait par étapes : premiers aménagements de la cavité par de vieilles abeilles exploratrices, construction de quelques cellules de réserve, transport de réserves de la vieille colonie vers la nouvelle, arrivée de jeunes abeilles en grand nombre, arrivée de la reine... Bref, lorsqu'on repère un nid dans une 
région, on a de fortes chances d'en trouver d'autres pas très loin : les nids sont assez souvent deux ou trois dans le même arbre; le cas le plus exceptionnel a été la découverte d'un arbre moyen contenant 22 colonies.

Tableau 2

Nombre d'arbres par catégorie dans chaque carré

Taberle 2

Zahl der Bäume je Klasse in jedem Abschnitt

\begin{tabular}{|c|c|c|c|c|c|}
\hline $\begin{array}{c}\text { Catégorie No } \\
\text { Klasse Nr } \\
\text { Carré } \mathbf{N o}^{\circ} \\
\text { Abschnitt Nr }\end{array}$ & 1 & 2 & 3 & 4 & $\begin{array}{c}\text { Nombre de } \\
\text { nids d'abeilles } \\
\text { Zahl der } \\
\text { Bienennester }\end{array}$ \\
\hline 1 & 7 & 5 & 2 & & 1 \\
\hline 2 & 7 & & 3 & & \\
\hline 3 & 15 & 6 & 1 & 1 & \\
\hline 4 & 29 & 5 & 2 & & 3 sur le même arbre \\
\hline 5 & 16 & 11 & 1 & & \\
\hline 6 & 8 & 5 & 1 & & \\
\hline 7 & 16 & 14 & 8 & & 1 \\
\hline 8 & 18 & 4 & 7 & & \\
\hline 9 & 14 & 10 & 5 & & \\
\hline 10 & 10 & 4 & 2 & & \\
\hline 11 & 8 & 7 & 5 & & \\
\hline 12 & 28 & 6 & 2 & & \\
\hline 13 & 27 & 7 & 3 & & \\
\hline 14 & 11 & 6 & 10 & & 1 \\
\hline \multicolumn{6}{|l|}{15} \\
\hline \multicolumn{6}{|l|}{16} \\
\hline \multicolumn{6}{|l|}{17} \\
\hline \multicolumn{6}{|l|}{18} \\
\hline \multicolumn{6}{|l|}{19} \\
\hline 20 & & & & & \\
\hline
\end{tabular}

Je possède le dénombrement des arbres dans 20 carrés de 30 mètres de côté dans un faciès arbustif dense. Ce nouvel exemple illustre encore sur une 
petite échelle les remarques précédentes. Les arbres ont été classés en 4 catégories suivant leur taille, $1^{\circ}$ ) ceux qui ne dépassent pas $2 \mathrm{~m}$, n'ont pas de grosses branches et de cœur creux, $2^{\circ}$ ) ceux qui ont une taille de 2 à $3 \mathrm{~m}$ et quelques branches, $3^{\circ}$ ) ceux qui mesurent de 4 à $5 \mathrm{~m}$, et enfin $4^{\circ}$ ) les grands arbres de forêt. Grosso modo la surface peut être représentée par un rectangle de 4 carrés sur 5 . Sur les 20 quadrats, 14 possèdent des arbres (Tableau 2).

Ainsi d'après ce tableau nous retrouvons sous une autre forme ce que nous avons déjà remarqué, à savoir l'indépendance totale qu'il y a entre la densité ou la taille des arbres (à condition toutefois qu'ils soient creux) et la densité des abeilles.

\section{VI. - RECHERChES SUR LA BIOLOGIE DES POPULATIONS D'ABEILLES}

Les abeilles construisent leurs rayons ou leurs cellules à couvain d'une manière spécifique. Les rayons des Dactylurina sont verticaux, ceux des Axestotrigones horizontaux ou hélicoidaux (l'hélice se développant dans un sens horizontal). Les petites cellules des Hypotrigones elles-mêmes présentent une orientation facilement identifiable. Elles sont ovales et leur plus grand axe est toujours perpendiculaire au sol. Cette remarque nous a permis de découvrir un moyen facile d'étudier la cadence de ponte en un temps donné sans traumatiser aucunement les colonies. En effet, il suffit lors de la récolte d'une colonie de repérer l'orientation de la branche qui le contient par rapport au sol. La branche est alors amenée sous un abri à proximité du laboratoire et orientée à $90^{\circ}$ par rapport à la direction qu'elle avait dans la nature. On laisse les abeilles libres de vaquer à leurs occupations pendant un temps donné, en suite de quoi on ouvre la ruche et l'on trouve les cellules nouvellement formées construites perpendiculairement à celles précédant la récolte (fig. 8). Il suffit alors de dénombrer les cellules ainsi reconnaissables pour savoir combien d'œufs ont été pondus. Cette expérience très facile à réaliser donne des indications précieuses quant au cycle biologique des abeilles en fonction de la saison, de l'importance de la colonie etc. Par ce moyen on peut aussi connaître la durée du développement larvaire et la productivité des colonies, le tout dans des conditions les plus naturelles possibles, la reine continuant à pondre normalement sans être perturbée.

Nos observations sont résumées dans le tableau 3. Ce tableau nous donne plusieurs sortes d'indications :

1) La durée de développement de l'ouvrière établie chez $H$. pothieri diffère légèrement de celle de l'ouvrière d'H. gribodoi Mayn déterminée par Bassindale (1955) dans des conditions expérimentales. Cet auteur estime à 35 jours le temps de développement d'une ouvrière. En Côte d'Ivoire, nous 


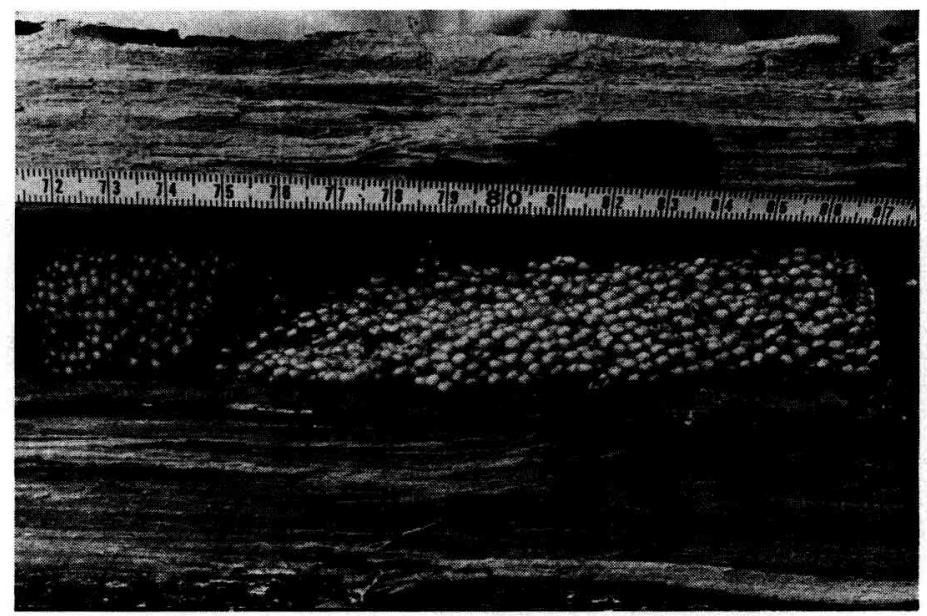

FIG. 8. - Couvain d'Hypotrigones dont les nouvelles cellules sont expérimentalement disposées dans une direction différente de celle des anciennes à la suite d'une rotation d'environ $90^{\circ}$ de la branche coupée contenant le nid d'abeilles.

Aвв. 8. - Brut von Hypotrigona, bei der die neuen Zellen experimentell in eine andere Richtung als die alten Zellen gebracht wurden, nachdem der abgeschnittene Ast mit dem Bienennest um etwa $90^{\circ}$ gedreht worden war.

avons constaté qu'un certain nombre de vieilles nymphes sont encore dans les cocons après 33 jours et que toutes sont écloses après 39 jours. Il semble donc, d'après les calculs sur la population non éclose restante et la ponte journalière de la reine, que dans notre cas particulier la durée du cycle soit voisine de 39 à 40 jours.

2) La ponte journalière d'une reine est assez variable d'une colonie à l'autre. Elle va de $22 \propto u f s$ à 82 environ. Cette constatation n'a rien d'étonnant pour ceux qui connaissent la biologie des reines d'Apis mellifica. Chez celle-ci en effet, la fécondité est très variable d'une reine à l'autre et est influencée aussi par l'état physiologique de l'animal considéré (âge, traumatismes éventuels, etc.).

3) Il apparaît enfin que le taux de ponte de la reine est indépendant du nombre d'individus présents dans la colonie. Si l'on rapporte à 100 ouvrières le taux de ponte quotidien de la reine on constate qu'il n'y a pas forcément corrélation entre ce taux de ponte et l'importance de la colonie. Une grosse colonie n'a donc pas obligatoirement une reine toujours plus fertile et inversement.

Notre étonnement a été grand de constater que la moyenne mensuelle des poids du couvain (estimés en grammes), reste à peu près la même durant 


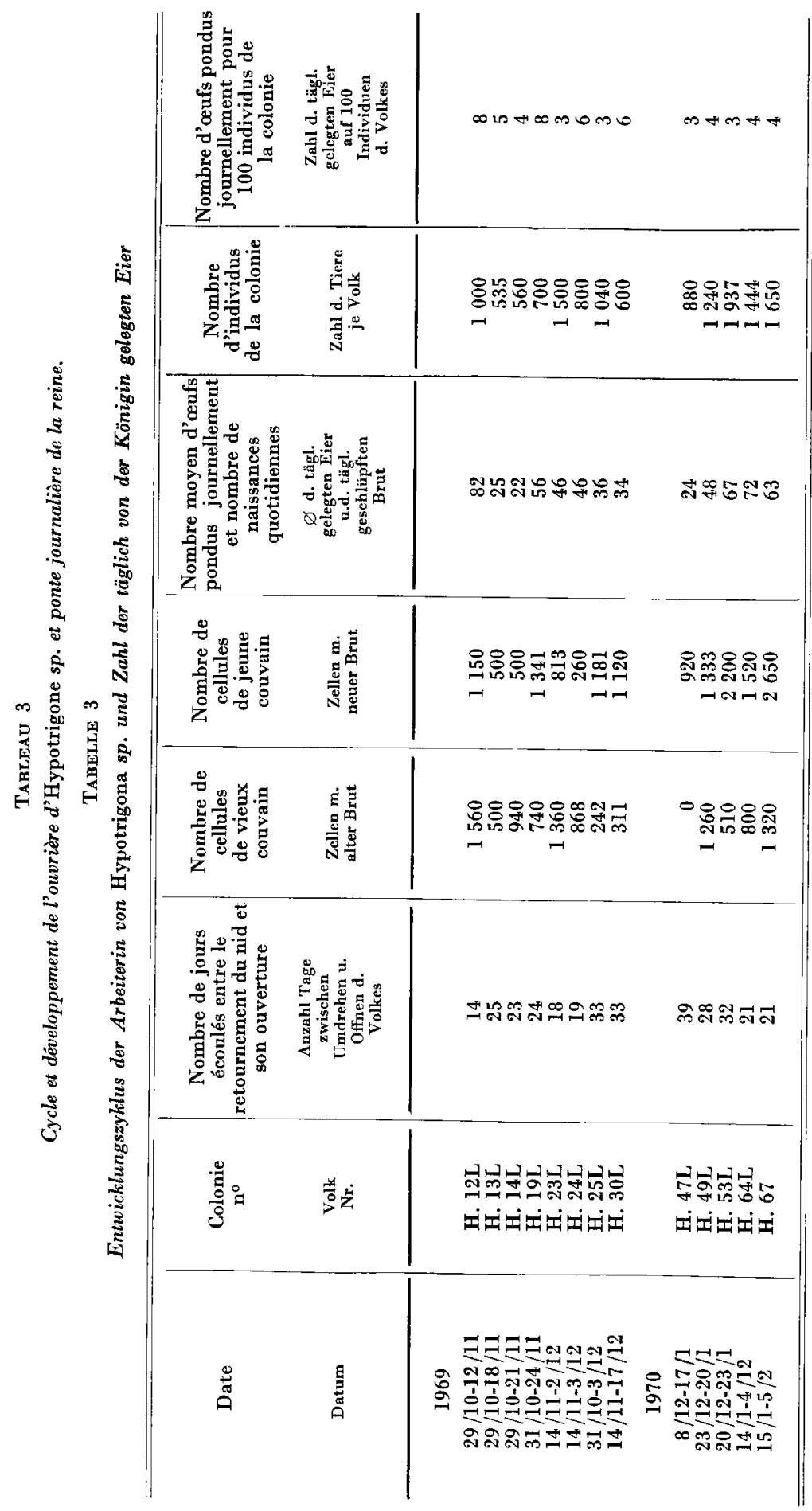


la saison des pluies et la saison sèche dans les diverses ruches que nous avons ouvertes en 1969 et 1970 (Tableau 4).

TABLEAU 4

Moyennes pondérales du couvain (jeune et vieux) présents dans les colonies (en grammes)

TABelle 4

Durchschnittsgewicht der in den Völkern vorhandenen Brut (jung und alt) in Gramm

\begin{tabular}{|c|c|c|c|c|c|}
\hline $\begin{array}{l}\text { Dates } \\
\text { Datum }\end{array}$ & $\begin{array}{c}\begin{array}{c}\text { Nombre } \\
\text { de }\end{array} \\
\text { colonies } \\
\\
\text { Zahl d. } \\
\text { Völker }\end{array}$ & $\begin{array}{c}\text { Moyenne } \\
\text { pondérale du } \\
\text { couvain au } \\
\text { moment de la } \\
\text { récolte des } \\
\text { colonies } \\
\varnothing \text { gewieht } \\
\text { d. Brut beim } \\
\text { Einfangen d. } \\
\text { Völker }\end{array}$ & $\begin{array}{c}\text { Moyenne } \\
\text { pondérale du } \\
\text { couvain } \\
\text { établies } \\
\text { mensuellement } \\
\text { Monatl. } \\
\varnothing \text { gewicht } \\
\text { d. Brut }\end{array}$ & $\begin{array}{c}\text { Nombre } \\
\text { d'individus } \\
\text { des groupes } \\
\text { de colonie } \\
\text { étudiées } \\
\text { Zahl d. } \\
\text { Tiere in } \\
\text { d. unter- } \\
\text { suchten } \\
\text { Gruppen }\end{array}$ & $\begin{array}{c}\text { Nombre } \\
\text { moyen } \\
\text { d'individus } \\
\text { d'une colonie } \\
\\
\varnothing \text { Zahl an } \\
\text { Individuen } \\
\text { eines } \\
\text { Volkes }\end{array}$ \\
\hline $\begin{array}{c}1968 \\
8 / 3-9 / 3 \\
19 / 3 \\
25 / 3-26 / 3 \\
1969-70\end{array}$ & $\begin{array}{l}4 \\
? \\
6\end{array}$ & $\begin{array}{r}12 \\
? \\
5\end{array}$ & 8 & & \\
\hline $\begin{array}{c}7 / 10-10 / 10 \\
11 / 10-29 / 10 \\
12 / 11-18 / 11 \\
21 / 11-25 / 11 \\
1 / 12-3 / 12 \\
13 / 12-19 / 12 \\
22 / 12-30 / 12 \\
5 / 1-9 / 1 \\
16 / 1-20 / 1 \\
21 / 1-31 / 1 \\
2 / 2-10 / 2\end{array}$ & $\begin{array}{l}4 \\
5 \\
2 \\
4 \\
5 \\
5 \\
6 \\
6 \\
3 \\
7 \\
8\end{array}$ & $\begin{array}{l}20 \\
12 \\
19 \\
17 \\
11 \\
11 \\
13 \\
13 \\
13 \\
31 \\
15\end{array}$ & $\begin{array}{l}16 \\
18 \\
12 \\
\\
19 \\
15\end{array}$ & $\begin{array}{rl}5 & 607 \\
2 & 676 \\
1 & 535 \\
2 & 335 \\
4 & 900 \\
3 & 480 \\
8 & 380 \\
8 & 530 \\
2 & 880 \\
13237 \\
11970\end{array}$ & $\begin{array}{r}1401 \\
535 \\
768 \\
584 \\
598 \\
980 \\
696 \\
1397 \\
1421 \\
960 \\
1891 \\
1496\end{array}$ \\
\hline
\end{tabular}

Nous pensions en effet que la ponte devait baisser notablement durant la saison sèche. En fait, même si les réserves de miel et de pollen diminuent sensiblement comme nous allons le voir, les Abeilles trouvent toujours à l'intérieur de leur habitation assez de nourriture entreposée pour alimenter normalement les larves. En effet, les Abeilles sociales amassent toujours plus de réserves qu'elles n'en utilisent : ainsi elles ne sont jamais à court d'aliments aux périodes de mauvaises récoltes. D'autre part, en Côte d'Ivoire, les minima thermiques ne sont en général jamais assez bas pour arrêter chez les ouvrières les activités de butinage et d'élevage du couvain et, chez la reine, l'activité de ponte.

Sachant qu'une cellule de jeune couvain, ou de vieux, pèse en moyenne $10 \mathrm{mg}$, qu'une nymphe ou qu'un œuf et sa nourriture pèsent $3 \mathrm{mg}$, on peut estimer à environ $5 \mathrm{~g}$ le poids des nymphes, des larves et de leur nourriture 
dans une colonie. Sachant aussi qu'on peut estimer à 1100 le nombre d'individus d'une colonie et qu'une ouvrière pèse $3 \mathrm{mg}$, le poids de matière vivante d'une colonie est donc environ $8,3 \mathrm{~g}$.

Si l'on se souvient enfin qu'on a découvert une moyenne de 2,5 nids à l'hectare, le poids de matière vivante d'Abeilles à l'hectare est donc environ $21 \mathrm{~g}$.

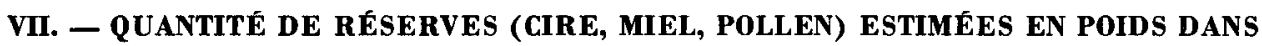 LES COLONIES D'HYPOTRIGONES AU COURS DE L'ANNEE}

Dans les nids de Trigones il est impossible de séparer les divers éléments des réserves (miel, pollen et cire) car tous sont imbriqués les uns dans les autres. L'extraction du miel par les indigènes est de ce fait restée extrêmement primitive. Les récoltes sont écrasées et mises dans des tissus à la chaleur. Le miel filtre ainsi dans des récipients entraînant du pollen et même des parcelles de cire. Devant l'impossibilité de séparer proprement les différentes sortes de réserves nous avons pesé tout l'ensemble en le détachant le plus délicatement possible des parois du nid (Tableau 5).

\section{TABLEaU 5}

Moyennes pondérales des réserves en cire, miel et pollen dans les colonies au cours des saisons

TABELLE 5

Durchschnittsgewicht von Wachs, Honig und Pollen in den Völkern im Verlauf der Jahreszeiten

\begin{tabular}{|c|c|c|c|c|}
\hline $\begin{array}{l}\text { Dates } \\
\text { Datum }\end{array}$ & $\begin{array}{l}\text { Nombre } \\
\text { de Colonies } \\
\text { Zahl d. } \\
\text { Völker }\end{array}$ & $\begin{array}{l}\text { Total } \\
\text { des réserves } \\
\text { en g. } \\
\text { Gesamtgewicht } \\
\text { d. Vorräte in } \\
\text { g }\end{array}$ & $\begin{array}{c}\text { Moyennes pondérales } \\
\text { des réserves au } \\
\text { moment de la récolte } \\
\text { des colonies } \\
\varnothing \text { gewicht d. } \\
\text { Vorrăte beim } \\
\text { Einfangen }\end{array}$ & $\begin{array}{c}\text { Moyennes pondérales } \\
\text { des réserves établies } \\
\text { mensuellement } \\
\varnothing \text { gewicht d. } \\
\text { monatl. ein- } \\
\text { getragenen } \\
\text { Vorräte }\end{array}$ \\
\hline 1968 & & & & \\
\hline $\begin{array}{c}8 / 3-9 / 3 \\
19 / 3 \\
25 / 3-26 / 3 \\
1969-70\end{array}$ & $\begin{array}{l}5 \\
2 \\
7\end{array}$ & $\begin{array}{r}175 \\
13 \\
106\end{array}$ & $\begin{array}{r}35 \\
7 \\
15\end{array}$ & 19 \\
\hline $\begin{array}{c}7 / 10-10 / 10 \\
11 / 10-29 / 10 \\
12 / 11-18 / 11 \\
21 / 11-25 / 11 \\
1 / 12-3 / 12 \\
13 / 12-19 / 12 \\
22 / 12-30 / 12 \\
5 / 1-9 / 1 \\
16 / 1-20 / 1 \\
21 / 1-31 / 1 \\
2 / 2-10 / 2\end{array}$ & $\begin{array}{r}4 \\
5 \\
2 \\
4 \\
5 \\
5 \\
6 \\
3 \\
3 \\
8 \\
10\end{array}$ & $\begin{array}{r}357 \\
297 \\
197 \\
297 \\
147 \\
223 \\
346 \\
62 \\
100 \\
352 \\
158\end{array}$ & $\begin{array}{l}89 \\
59 \\
99 \\
74 \\
29 \\
45 \\
58 \\
21 \\
33 \\
44 \\
16\end{array}$ & $\begin{array}{r}327 \\
247 \\
\\
239\end{array}$ \\
\hline
\end{tabular}


La colonne des moyennes mensuelles des poids fait apparaître une diminution notable des réserves durant 6 mois, période qui va de la saison des pluies (octobre-décembre) à la saison sèche bien entamée (janvier-mars). Il serait d'ailleurs intéressant de poursuivre cette expérience au moins jusqu'à la fin de la saison sèche en Côte d'Ivoire. Cette diminution est spectaculaire car la chute est d'environ $230 \mathrm{~g}$ en 3 mois. La consommation des réserves pendant la saison sèche est donc impressionnante mais elle ne nous étonne pas, puisque l'on sait que les élevages de couvain continuent normalement et que les Abeilles doivent aussi se nourrir sur ces réserves car il y a pénurie de nourriture produite par la végétation à cette époque.

A Makokou (Gabon), en 1968, nous avions déjà commencé un certain nombre d'expériences assez semblables et dont les résultats vont dans le sens précédent. Ils viennent à point ici pour compléter les observations que nous venons de faire.

Dans cette ville entourée de forêts, nous possédons un véritable rucher composé en grande partie de différentes espèces d'Hypotrigones élevées soit dans des boîtes en matière plastique transparente, soit dans des ruchettes vitrées très plates. Il est donc très facile de voir ce qui se passe à l'intérieur des colonies et de peser régulièrement chacune d'entre elles. Les premières pesées ont été effectuées en avril et les dernières en juillet, c'est-à-dire commencées à la fin de la petite saison des pluies et terminées au milieu de la grande saison sèche. Or cette année-là, nous avons eu la chance d'avoir quelques nouveaux essaims à une période tardive. Ils sont en effet très nombreux durant les deux premiers et le dernier mois de l'année. Nous avons établi deux tableaux l'un concernant les anciennes populations (Tableau 6) et l'autre les nouvelles (Tableau 7).

$\left.1^{\circ}\right)$ Après avoir augmenté régulièrement durant deux mois (saison des pluies) le poids de chaque colonie décroît régulièrement jusqu'à la fin des expériences (saison sèche). Les résultats sont comparables à ceux de la Côte d'Ivoire.

$2^{\circ}$ ) Cependant la diminution du poids durant la saison sèche est lente et non plus rapide. Évidemment des expériences seraient nécessaires pour comprendre ce phénomène différent. On peut toutefois en imaginer les causes : dans la région où sont menées ces expériences la température descend notablement durant la nuit; le jour, le ciel est gris. En saison sèche, il fait donc un froid relatif amenant chez les abeilles une sorte d'hibernation. Elles élèvent moins de couvain, butinent moins mais consomment moins aussi, les colonies menant véritablement une vie ralentie à ce moment-là, la chute des réserves est de ce fait moins spectaculaire.

Il est en revanche étonnant de constater que pendant la même période de l'année le poids des nouvelles colonies augmente régulièrement. Ce phéno- 
mène rappelle le comportement des nouveaux essaims de nos Abeilles domestiques (Apis mellifica) qui en quelques jours construisent plusieurs rayons de cire, amassent du pollen et du miel dans des proportions infiniment plus grandes que celles des colonies établies dans leurs ruches depuis plusieurs mois. Chez les Trigones, à cela s'ajoute le fait que les colonies filles sont en général en relation constante avec la colonie mère et qu'il leur est loisible d'emprunter des réserves à celle-ci pour constituer les leurs. Ici le phénomène est plus frappant que chez Apis mellifica car cette augmentation de poids des jeunes colonies correspond au même moment à une diminution générale des réserves pour les colonies âgées.

Tableau 6

Variation de poids de jeunes colonies d'Hypotrigones sp. (en grammes)

TABeLLE 6

Gewichtsveränderung junger Völkchen von Hypotrigona in Gramm

\begin{tabular}{|c|c|c|c|c|c|c|}
\hline $\begin{array}{c}\text { Colonie } \\
\text { Völker } \\
\text { date } \\
\text { des pesées } \\
\text { Tag d. } \\
\text { Wägung }\end{array}$ & 1 & 2 & 3 & 4 & $\begin{array}{c}\text { Total } \\
\text { Gesant- } \\
\text { gewicht }\end{array}$ & $\begin{array}{c}\begin{array}{c}\text { Moyenne } \\
\text { en }\end{array} \\
\text { grammes } \\
\begin{array}{c}\text { Durchschnitt } \\
\text { in } \mathrm{g}\end{array}\end{array}$ \\
\hline $1 / 5 / 68$ & 39 & 30,7 & & & & 35 \\
\hline $8 / 5$ & 41,8 & 32 & 29 & 36,5 & 139 & 34 \\
\hline $16 / 5$ & 44,9 & 35 & 29,5 & 38,3 & 148 & 37 \\
\hline $25 / 5$ & 45,8 & 36,8 & 29,5 & 39 & 148 & 37 \\
\hline $4 / 6 / 68$ & 46,8 & 39,8 & 29,5 & 40,2 & 156 & 39 \\
\hline $12 / 6$ & 47,3 & 42,1 & 29,5 & 40,6 & 160 & 40 \\
\hline $22 / 6$ & 47,6 & 44,1 & 29,9 & 41,7 & 163 & 40 \\
\hline $9 / 7 / 68$ & 48 & 45,1 & 31 & 42 & 166 & 41 \\
\hline
\end{tabular}

Il reste à évaluer le poids des réserves des Hypotrigones sur un hectare. Il est évident maintenant que l'on doit connaître la saison pendant laquelle on effectue le calcul. Au mois d'octobre, on peut l'estimer à $820 \mathrm{~g}$ alors qu'en mars il ne dépasse pas $50 \mathrm{~g}$. D'une façon comme d'une autre, il s'agit toujours quand même de très faibles quantités. 


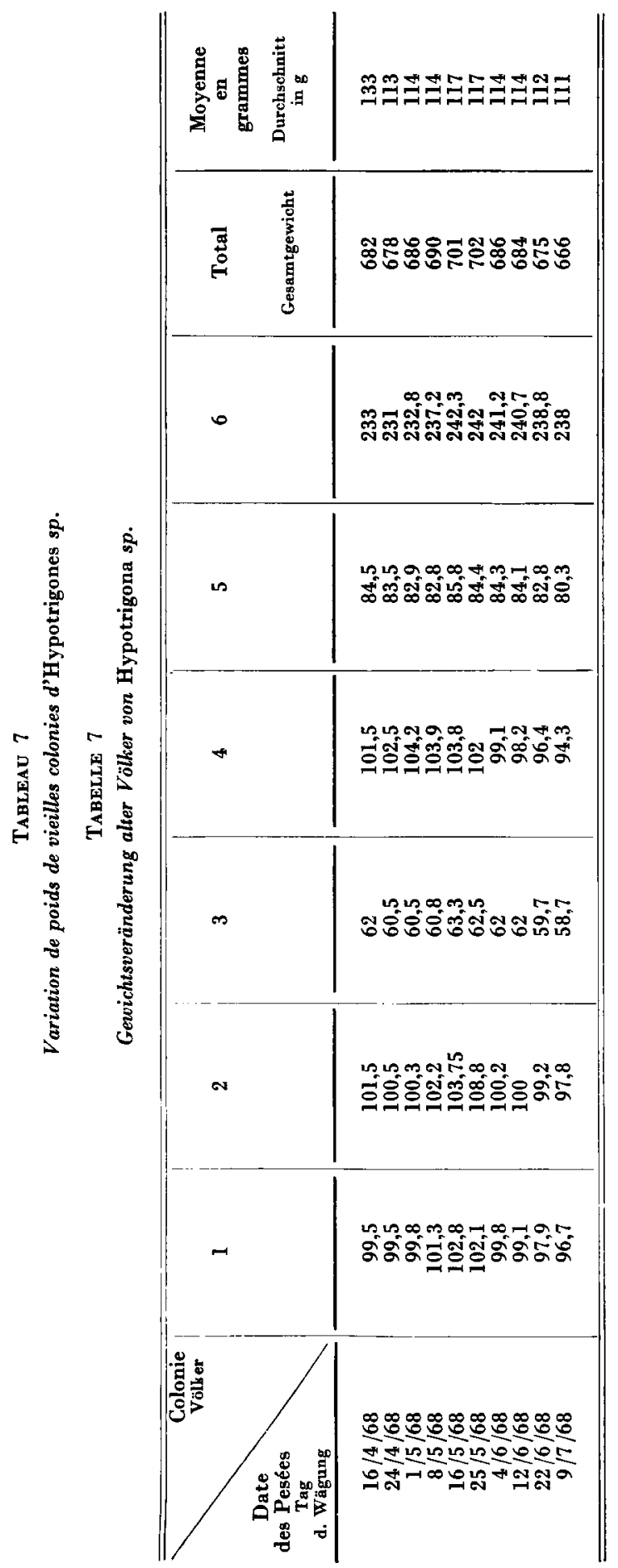




\section{VIII. - L'ACTivité DE BUtinage ET LA POLlinisation Chez LES TRIgones}

Nous n'avons pas effectué de mesures d'activité avec les Hypotrigones africaines de Lamto (savanes de Côte d'Ivoire). Toutefois nous pouvons insérer ici des résultats obtenus à Makokou (région de forêt au Gabon) et cela pour deux raisons, $1^{\circ}$ ) d'abord, parce que les colonies d'Hypotrigones étudiées au Gabon étaient situées dans un endroit dégagé, lumineux, à proximité d'une petite ville aux maisons clairsemées, et non pas en forêt obscure. On peut donc, sans trop de risques d'erreur, comparer l'activité des animaux dans ce biotope à celle des mêmes abeilles vivant en savane. $2^{\circ}$ ) ensuite, parce que des tests d'activité menés au Cameroun pour étudier la pollinisation des cacaoyers en région de savane ont donné des résultats comparables à ceux obtenus au Gabon (de MIRE, communication orale, 1971).

Voici donc les comptages effectués à Makokou à l'extrémité du tube de sortie d'un nid d'Hypotrigones durant la petite saison des pluies (mars-mai) et durant la première moitié de la saison sèche (juin-juillet) en 1967 et 1968 (Tableau 8).

Tableau 8

L'activité de butinage des Hypotrigones au Gabon en 1968 entre $11 \mathrm{~h}$ et $12 \mathrm{~h}$

Tabelle 8

Sammeltätigkeit der Hypotrigonen in Gabun 1968 zwischen 11 und 12 Uhr mittags

\begin{tabular}{|c|c|c|c|c|c|c|c|}
\hline Date & $\begin{array}{c}\text { Température } \\
\text { o C } \\
\begin{array}{c}\text { Eelairement } \\
\text { lux }\end{array} \\
\text { Temperatur } \\
\text { 0 C } \\
\text { Helligkeit } \\
\text { Lux }\end{array}$ & $\begin{array}{l}\text { Sorties } \\
\text { Ausflïge }\end{array}$ & $\begin{array}{l}\text { Entrées } \\
\text { Rüickflüge }\end{array}$ & $\begin{array}{l}\text { Date } \\
\text { Datum }\end{array}$ & $\begin{array}{c}\text { Température } \\
\text { OC } \\
\text { Éclairement } \\
\text { lux } \\
\text { Temperatur } \\
0 \text { C } \\
\text { Helligkeit } \\
\text { Lux }\end{array}$ & $\begin{array}{l}\text { Sorties } \\
\text { Ausflüge }\end{array}$ & $\begin{array}{l}\text { Entrées } \\
\text { Rückflüge }\end{array}$ \\
\hline $\begin{array}{c}20 / 4 / 68 \\
11 / 5 \\
15 / 5 \\
17 / 5 \\
20 / 5 \\
25 / 5 \\
28 / 5 \\
30 / 5 \\
1 / 6 \\
3 / 6 \\
6 / 6 \\
7 / 6 \\
10 / 6 \\
11 / 6 \\
12 / 6 \\
13 / 6\end{array}$ & $\begin{array}{c}\text { couvert } \\
25^{0} 5 ; 2500 \\
26^{0} 5 ; 1850 \\
28^{\circ} 5 ; 3200 \\
28^{\circ} 5 ; 3100 \\
27^{0} 5 ; 2000 \\
27^{\circ} 5 ; 2850 \\
27^{\circ} 5 ; 3000 \\
27^{0} 5 ; 4000 \\
27^{\circ} ; 2000 \\
27^{0} 5 ; 4800 \\
27^{0} 5 ; 3800 \\
27^{\circ} 5 ; 1200 \\
27^{0} 5 ; 2200 \\
26^{\circ} ; 1750 \\
27^{0} ; 2000\end{array}$ & $\begin{array}{r}140 \\
31 \\
169 \\
321 \\
138 \\
209 \\
185 \\
287 \\
248 \\
139 \\
242 \\
146 \\
77 \\
303 \\
109 \\
130\end{array}$ & $\begin{array}{r}112 \\
33 \\
150 \\
285 \\
187 \\
193 \\
156 \\
223 \\
240 \\
158 \\
220 \\
181 \\
77 \\
287 \\
115 \\
140\end{array}$ & $\begin{array}{l}17 / 6 \\
19 / 6 \\
20 / 6 \\
21 / 6 \\
24 / 6 \\
26 / 6 \\
27 / 6 \\
28 / 6 \\
29 / 6 \\
1 / 7 \\
2 / 7 \\
3 / 7 \\
5 / 7 \\
6 / 7 \\
8 / 7\end{array}$ & $\begin{array}{l}27^{0} ; 2500 \\
26^{0} 5 ; 2500 \\
27^{0} ; 2500 \\
26^{0} ; 2400 \\
24^{0} ; 1200 \\
24^{0} 5 ; 2400 \\
25^{0} ; 1200 \\
25^{0} 5 ; 2000 \\
27^{0} 5 ; 3300 \\
24^{0} 5 ; 3200 \\
24^{0} ; 1200 \\
23^{0} ; 1200 \\
23^{0} 5 ; 1200 \\
23^{0} ; 1200 \\
27^{0} ; 3700\end{array}$ & $\begin{array}{r}183 \\
201 \\
259 \\
140 \\
44 \\
92 \\
92 \\
133 \\
189 \\
68 \\
126 \\
54 \\
72 \\
88 \\
68\end{array}$ & $\begin{array}{r}203 \\
215 \\
240 \\
148 \\
42 \\
82 \\
96 \\
130 \\
130 \\
65 \\
146 \\
48 \\
51 \\
87 \\
60\end{array}$ \\
\hline
\end{tabular}


Le tableau 9 tente de donner un résumé du précédent alors que le tableau 10 présente les moyennes mensuelles des sorties d'un nid d'Hypotrigones, les moyennes mensuelles des températures et des éclairements durant la même période de temps.

Tableau 9

Moyennes mensuelles des sorties ot des entrées entre 11 h et 12 h d'un nid d'Hypotrigone. Moyennes mensuelles des températures et des éclairements durant la même période de temps.

\section{TABELLE 9}

Monatlicher Durchschnitt der Aus-und Rückflüge zwischen 11 und 12 Uhr in einem Hypotrigonen-Nest. Monatsmittel der Temperaturen und der Helligkeit in der gleichen Zeitspanne.

\begin{tabular}{|c|c|c|c|c|c|}
\hline $\begin{array}{c}\text { Saison } \\
\text { Jahreszeit }\end{array}$ & $\begin{array}{l}\text { Mois } \\
\text { Monat }\end{array}$ & $\begin{array}{l}\text { Sorties } \\
\text { Ausflüge }\end{array}$ & $\begin{array}{l}\text { Moyenne horaire } \\
\text { du mois } \\
\text { Monatl. } \\
\text { Stundendurebschnitt }\end{array}$ & Lux & $\begin{array}{c}\mathrm{T}^{0} \text { moyenne } \\
\text { Durchsehnitts- } \\
\text { temperatur }\end{array}$ \\
\hline $\begin{array}{c}\text { des pluies } \\
\text { Regen }\end{array}$ & $\begin{array}{r}\text { mai } \\
\text { Mai }\end{array}$ & 1340 & 191 & 2643 & $27^{\circ} 1 / 4$ \\
\hline $\begin{array}{l}\text { sèche } \\
\text { trocken }\end{array}$ & $\begin{array}{l}\text { juin } \\
\text { Juni }\end{array}$ & 1394 & 174. & 2719 & $27^{\circ} 1 / 5$ \\
\hline- & $\begin{array}{c}\text { juillet } \\
\text { Juli }\end{array}$ & 1333 & 148 & 2222 & $26^{\circ}$ \\
\hline- & $\begin{array}{c}\text { août } \\
\text { August }\end{array}$ & 476 & 79 & 1950 & $24^{0} 1 / 5$ \\
\hline
\end{tabular}

On voit ainsi que le nombre des sorties durant la même heure, de 11 heures à midi, diminue depuis la petite saison des pluies jusqu'au milieu de la saison sèche. Ce phénomène est en relation évidente avec la baisse de température moyenne et l'éclairement. Il faut ajouter immédiatement que ces deux facteurs ne sont pas exclusifs d'autres facteurs qui leur sont liés intimement comme la floraison, la production du pollen.

Enfin avec un dernier tableau (11) on aura une idée de l'ampleur de l'activité de butinage d'une colonie durant une journée normale à Makokou (Gabon).

Il y a ainsi en moyenne par nid 800 sorties d'Abeilles par jour durant l'année. Au-dessus d'un hectare de savane de Lamto, les Hypotrigones effectuent vraisemblablement environ 2000 vols journaliers; elles visitent donc des milliers de fleurs. 
TABLEAU 10

TABelLe 10

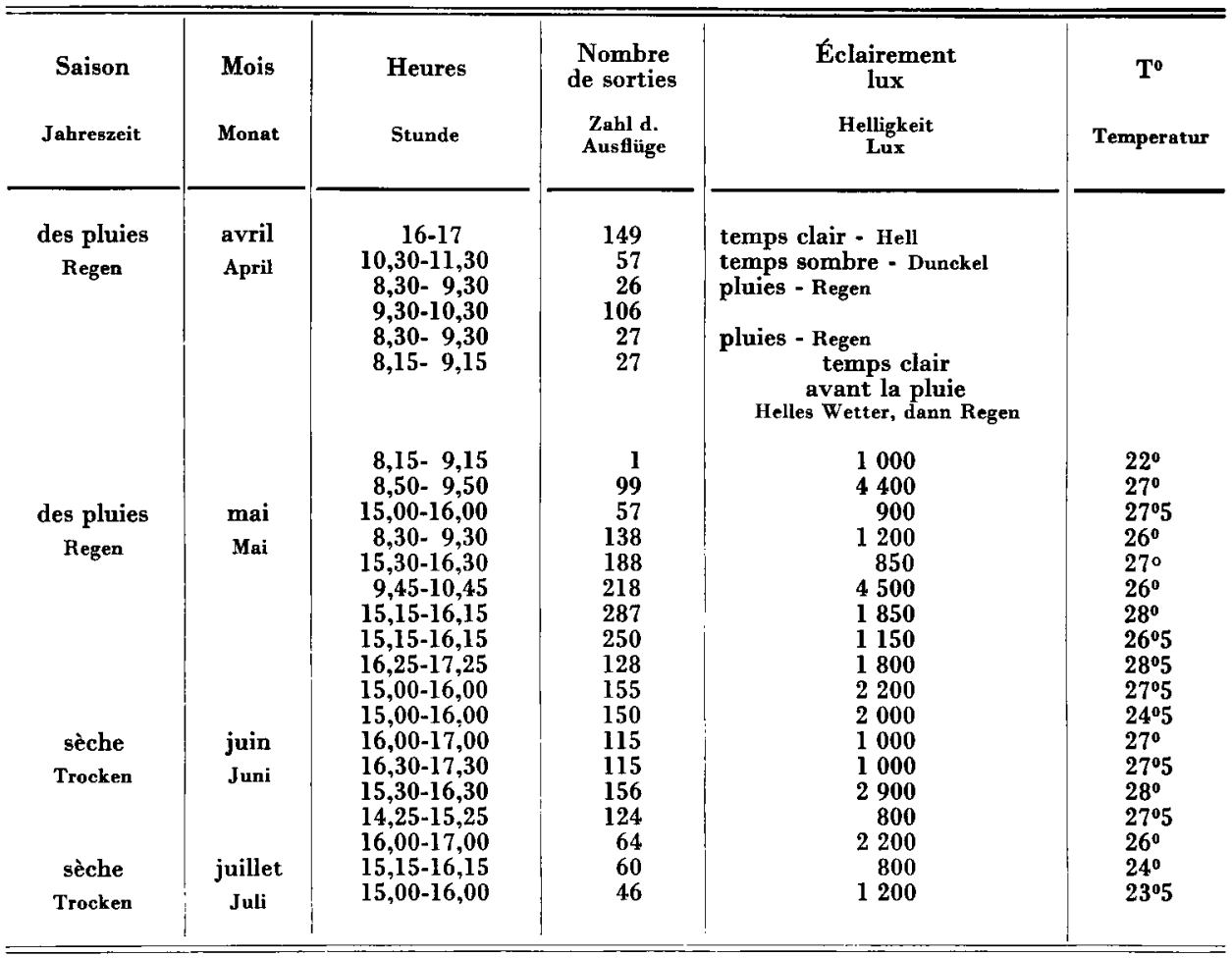

Ajoutons encore qu'à côté des Hypotrigones il y a dans la savane quelques nids d'Axestotrigones contenant environ 2000 individus, de Dactylurines de 20000 insectes et, dans la forêt galerie, des nids d'autres Trigones et d'Apis mellifica adansonii que l'on rencontre fréquemment sur les graminées de la savane.

\section{IX. - CONCLUSION}

Comment expliquer le petit nombre de colonies à l'hectare alors que les arbres creux sont innombrables dans la savane? Je crois qu'ici il faut faire intervenir la compétition entre les Fourmis arboricoles et les Abeilles. D'après Delage-Darchen (1971) on sait maintenant très bien qu'une grande partie des cavités des arbres de plus de $10 \mathrm{~cm}$ de diamètre sont occupées par des Fourmis Crematogaster. 
Tableau 11

Activité des Abeilles aux différentes heures de la journêe durant le mois d'avril

TABetLe 11

Tätigkeit der Bienen zu verschiedenen Tageszeiten im April

\begin{tabular}{c|c}
\hline \hline Heure & $\begin{array}{c}\text { Moyenne des sorties } \\
\text { sur un nombre de jours } \\
\text { variant de 2 à 9 } \\
\text { Stunde } \\
\text { Durchschnitt1. Ausfiuge } \\
\text { sährend einer zwischen 2 u.9 Tagen } \\
\text { wechselnden Zahl v. Tagen }\end{array}$ \\
\hline $9,30-9,00-9,30$ & 22 \\
$10,00-10,30$ & 27 \\
$11,00-11,30$ & 81 \\
$11,30-12,00$ & 80 \\
$14,30-15,00$ & 93 \\
$15,00-15,30$ & 62 \\
$15,30-16,00$ & 73 \\
$16,00-16,30$ & 75 \\
$16,30-17,00$ & 69 \\
\hline
\end{tabular}

Il n'y a pas de lutte active entre Fourmis et Abeilles, habitant dans un même arbre, ou dans une même cavité car les colonies sont séparées par une paroi de carton ou de résine et elles peuvent cohabiter en s'ignorant. Cependant, il y a quand même une compétition entre ces Insectes du fait qu'ils occupent les mêmes niches écologiques. Les Fourmis sont plus fécondes, plus nombreuses et plus agressives et par là-même ont plus de chance d'occuper les loges vacantes. Enfin les abeilles n'essaiment en général qu'à quelques mètres ou quelques dizaines de mètres de la ruche mère : il leur faut donc trouver une habitation à proximité, ce qui n'est pas souvent possible (DARCHEN, 1969). Elles arrivent ainsi quelquefois par hasard à coloniser toutes les cavités d'un même arbre, témoin l'arbre aux 22 nids d'Hypotrigones.

Que sont enfin 2750 Hypotrigones, que représentent 21 g de matière vivante (larves + imagos) et quelques centaines de grammes de miel et de pollen à l'hectare de savane en face de la biomasse des Cérambycides, des vers... ou même d'insectes sociaux comme les Termites ou les Fourmis? Presque rien.

En fait, il apparaît bien maintenant que le véritable problème écologique des abeilles sociales soit un problème de pollinisation. Il est évident que la biomasse, le biovolume et le poids sec des abeilles n'a presqu'aucun sens ici, 
mais que les notions de travail, de joules devraient être plus familières à l'apidologue écologiste.

Cette conclusion à elle seule trace le futur plan de travail.

Reçu pour publication en mars 1972

Eingegangen im März 1972

\section{REMERCIEMENTS}

Je remercie M. BARBault pour m'avoir fourni le dénombrement précis des arbres dans 20 carrés de savane arbustive.

\section{ZUSAMMENFASSUNG}

Mit dieser Arbeit wird versucht, die ökologische Bedeutung der sozialen Bienen, besonders der Trigonen, in der Savanne von Lamto, Elfenbeinküste zu erhellen.

Lage und Biotop. Die Forschungsstelle für tropische Ökologie in Lamto befindet sich etwa $200 \mathrm{~km}$ nordöstlich von Abidjan in einer Savanne, die mit ihren Borassuspalmen (Borassus aethiopum) typisch für Guinea ist. Die Savanne besteht aus einem Gräserteppich, in dem Bäume und auch Sträucher wachsen. Vor allem fünf der am häufigsten vorkommenden Bäume bieten Innenhohlräume, die hauptsächlich von Ameisen (Crematogaster) und Bienen zum Nestbau benutzt werden. Während die Ameisen lebende Bäume bevorzugen, bewohnen die Bienen (Hypotrigonen, Liotrigonen) ebenso gern abgestorbene Bäume.

In der Savanne gibt es auch Bienen, die nicht in Bäumen nisten. Einige wählen die Hohlräume in grossen Termitenhügeln, andere errichten mit Hilfe von Harz und Wachs vollkommen freigebaute Nester (Dactylurinen).

Die Bienengattungen der Savanne. Nur fünf Vertreter von den zehn jetzt in Afrika bekannten Gattungen konnten wir finden, und zwar höchstens ein Dutzend Arten, von denen drei oder vier bisher unbekannt waren. $Z u$ beachten ist auch, dass neben den in der Savanne gefangenen Arten einige aus den stets in der Nähe vorhandenen Galeriewäldern stammten (z.B. : Apis mellifica, Meliponula bocandei).

Einige Arten scheinen besondere Fähigkeiten zur Wärmeregulierung zu besitzen.

Einfluss der Savannenbrände auf die Bienen. Es hat sich erwiesen, dass die meisten Nester trotz der Feuersbrunst unbeschädigt bleiben. Allerdings schmilzt in den Nestern, die verhältnismässig tief in den Bäumen angelegt sind, das Wachs der Brutzellen, und die Brut stirbt ab. Zur Zeit der Savannenbrände gibt es auch Verluste in der Volksentwicklung gewisser Bienenvölker, obwohl die Königin und eine grössere Zahl von Arbeiterinnen am Leben bleiben.

Ausserdem zerstört das Feuer einen Grossteil der Nahrung, die die krautigen Pflanzen der Savanne den Bienen liefern. Es scheint daher, dass das Feuer zusammen mit der Trockenperiode zur Verringerung der Futterreserven und der Brut beiträgt, die um diese Zeit zu beobachten ist.

Arbeitsmethode zur Schätzung der Dichte der Bienenpopulationen. Es wurde die klassische Methode des Auszählens der Nester auf willkürlich ausgewählten Flächen bekannter Grösse angewandt (zwischen $1 / 8$ ha und 1 ha). 
Ergebnisse. 1. Auf den $30 \mathrm{Ha}$, die in verschieden grosse Quadrate aufgeteilt waren, wurden durchschnittlich 2,5 Nester je Ha gefunden. (Nach LeviEuX gibt es 7000 Ameisennester und nach Josens 17 Millionen Termiten je ha). In manchen Gebieten gibt es gar keine, in anderen mehrere Nester.

2. Nicht jede Erscheinungsform der Savanne scheint ein Vorzugsbiotop für die Ansiedlung kleiner Bienenarten zu sein (Hypotrigonen und Liotrigonen).

3. Die Entwicklungsdauer der Arbeiterin von Hypotrigona beträgt $39-40$ Tage.

4. Die täglich von einer Königin abgelegte Zahl von Eiern ist in den einzelnenVölkchen verschieden, sie schwankt zwischen 22 und 82 Eiern.

5. Die Zahl der gelegten Eier ist unabhängig von der Individuenzahl eines Volkes.

6. In Lamto ist während der Regen- und der Trockenzeit der monatliche Durchschnitt des Brutgewichtes nahezu gleich.

7. Man kann annehmen, dass in einem mittelstarken Volk von 1100 Individuen, das $3,3 \mathrm{~g}$ wiegt, das Gewicht der Puppen, Larven und des Futters etwa $5 \mathrm{~g}$ beträgt, so dass ein Völkchen durchschnittlich 8,3 $\mathrm{g}$ und die 2,5 Völker je Ha demnach $21 \mathrm{~g}$ wiegen.

8. Zwischen Regen- und Trockenperiode nehmen die Futtervorräte beachtlich ab. Durchschnittlich betragen sie bei Hypotrigona im Oktober $820 \mathrm{~g}$ und im Mai $50 \mathrm{~g}$.

Was bedeuten 2750 Hypotrigonen je Ha Savanne angesichts der Menge von Cerambiden, Würmern oder auch anderer sozialer Insekten? Nahezu nichts! Und doch sind in anbetracht der etwa 800 täglichen Ausflüge eines Hypotrigona-Völkchens - das sind 2000 Sammelflüge auf einen Ha Savanne - die Bienen von überragender Bedeutung für die Savanne.

\section{REFÉRENCES BIBLIOGRAPHIQUES}

Bassindale R., 1955. The biology of the stingless bee, Trigona (Hypotrigona) gribodoi Mag., (Meliponidae) Proc. Zool. Soc. London 125, 1, 49-62.

Darchen R. 1969. La biologie des Trigones et des Melipones (Hyménoptères Apidae) à la lumière des travaux récents $A$ nn. Biol., 8, 7-8, 455-490.

Darchen R. 1969. Sur la biologie de Trigona (Apotrigona) nebulata Komiensis Cock. Biol. Gab., 5, 3, 151-187.

Darchen R. 1970. Le nid de deux nouvelles espèces d'Abeilles de la Côte d'Ivoire, Trigona (Axestotrigona) eburnensis Darchen et Trigona (Axestotrigona) sawadogoi Darchen (Hymen., Apidae). Biol. Gab., 6, 2, 139-150.

Delage-Darchen B. 1971. Écologie des Fourmis arboricoles de la savane de Lamto (Côte d'Ivoire). Biol. gabonica, (sous presse).

Josens G. 1969. Communication personnelle.

LevieuX J., 1969. Recherches écologiques dans la savane de Lamto (Côte d'Ivoire) : données préliminaires sur le peuplement en Fourmis terricoles. La terre et la Vie, 21, 278-296.

Mire (DE) B. Ph. 1971. Communication personnelle. 